\title{
DETAILED AND SIMPLIFIED NONEQUILIBRIUM HELIUM IONIZATION IN THE SOLAR ATMOSPHERE
}

\author{
Thomas Peter Golding, Mats Carlsson, and Jorrit LeenaArts ${ }^{1}$ \\ Institute of Theoretical Astrophysics, University of Oslo, P.O. Box 1029 Blindern, NO-0315 Oslo, Norway; thomas.golding@astro.uio.no, \\ mats.carlsson@astro.uio.no, jorritl@astro.uio.no \\ Received 2013 November 20; accepted 2014 January 27; published 2014 February 28
}

\begin{abstract}
Helium ionization plays an important role in the energy balance of the upper chromosphere and transition region. Helium spectral lines are also often used as diagnostics of these regions. We carry out one-dimensional radiation-hydrodynamics simulations of the solar atmosphere and find that the helium ionization is set mostly by photoionization and direct collisional ionization, counteracted by radiative recombination cascades. By introducing an additional recombination rate mimicking the recombination cascades, we construct a simplified three-level helium model atom consisting of only the ground states. This model atom is suitable for modeling nonequilibrium helium ionization in three-dimensional numerical models. We perform a brief investigation of the formation of the He I 10830 and He II 304 spectral lines. Both lines show nonequilibrium features that are not recovered with statistical equilibrium models, and caution should therefore be exercised when such models are used as a basis for interpretating observations.
\end{abstract}

Key words: line: formation - radiative transfer - Sun: atmosphere - Sun: chromosphere

Online-only material: color figures

\section{INTRODUCTION}

The gas in the solar atmosphere goes from mostly neutral in the photosphere to highly ionized in the corona. In the dynamic interface of these two regimes, the chromosphere and transition region, atoms will ionize and recombine on various timescales. The ionization will be out of equilibrium if the ionization/ recombination timescale is longer than the typical hydrodynamic timescale. The ionization balance of sufficiently abundant atomic species affects the energetics of the atmosphere, as an ionized atom stores energy that would otherwise increase the temperature of the gas. Stellar atmosphere simulations that use the simplifying assumption of statistical equilibrium (SE) might therefore miss potential effects of nonequilibrium ionization.

In a numerical model of the solar atmosphere, treating ionization-recombination processes in detail requires solving the complete radiative transfer problem - a nonlinear, nonlocal problem that three-dimensionally is too computationally demanding for present day computers to handle. In simpler geometry, however, the situation is different: Carlsson \& Stein $(1992,1995,1997)$ carried out one-dimensional simulations of a dynamic solar atmosphere where the nonequilibrium ionization and recombination of abundant elements was included, and they found that the effects of this are important for the thermodynamic structures of the atmosphere. In a follow-up study, it was shown that the relaxation timescales of hydrogen ionization and recombination are long compared with the dynamic timescales, especially in the cool postshock phase (Carlsson \& Stein 2002, hereafter referred to as CS2002). This leads to a lower ionization degree in chromospheric shocks and a higher ionization degree between the shocks, compared with the SE solution, because the hydrogen populations do not have time to adjust to the rapidly changing conditions.

On the basis of a simplified method for treating the radiative transition rates of hydrogen (Sollum 1999), an experiment with hydrogen ionization was carried out by Leenaarts \&

\footnotetext{
1 Now at Institutet för Solfysik, Stockholms Universitet.
}

Wedemeyer-Böhm (2006) in three dimensions. This study confirmed that hydrogen is out of equilibrium in the chromosphere. The experiment was repeated by Leenaarts et al. (2007) in two dimensions, but this time the ionization was included also in the equation-of-state, resulting in larger temperature variations in and between the shocks propagating in the chromosphere than what was found with an equation-of-state assuming local thermodynamic equilibrium (LTE). Nonequilibrium formation of $\mathrm{H}_{2}$ was later included in this method (Leenaarts et al. 2011) and is currently a part of the Bifrost stellar atmosphere code (Gudiksen et al. 2011).

Leenaarts et al. (2011) pointed out that their model had a temperature plateau $(\sim 10 \mathrm{kK})$ in the upper chromosphere and that it most likely was associated with the LTE treatment of helium in their equation-of-state. Our goal is to realistically treat the nonequilibrium ionization of both hydrogen and helium in Bifrost. With such a model, we plan to perform studies of the formation of the spectral lines He I 10830 (formed in the chromosphere) and He II 304 (formed in the transition region), both of which are often used diagnostics, e.g., SDO/AIA (Lemen et al. 2012), STEREO's SECCHI/EUVI (Howard et al. 2008), VTT/TIP II: (Collados et al. 2007), NST/NIRIS (Cao et al. 2012).

The 10830 line is an absorption line that forms when continuum photons from the photosphere are scattered or absorbed in the chromosphere by neutral helium atoms occupying the metastable $2 s^{3} S$ state. This state is populated mostly by recombination cascades that follow from the photoionization of neutral helium atoms by the coronal extreme ultraviolet (EUV) lithography incident radiation (Avrett et al. 1994; Mauas et al. 2005; Centeno et al. 2008). For this reason, the line maps out the boundaries of coronal holes, which are regions where the coronal EUV emission is weaker (Sheeley 1980; Harvey \& Recely 2002). He I 10830 is a valuable line also for studying active regions where the EUV emission is strong. An example is Ji et al. (2012), who used high-resolution imaging data from the New Solar Telescope (NST) to study heating events in small-scale 
magnetic loops. Another application is the study of magnetic fields: Xu et al. (2012) used inversions of the full Stokes vectors of the photospheric Si I 10827 and the chromospheric He I 10830 to study the magnetic field associated with an active region filament. Owing to its diagnostic potential, the 10830 line is one of the candidate lines for the planned Solar- $C$ mission.

He II 304 is an optically thick line that forms in the transition region. It is an important source of the impinging EUV radiation absorbed by the chromosphere; hence, it might be important for the spectrum of He I (Andretta et al. 2003) and for the energy balance of the chromosphere and transition region. The formation of He II 304 is still debated. As noted by Jordan (1975), the helium-line intensities are larger than what is predicted by models constructed from observations of other EUV lines. Jordan introduced the idea of high-energy electrons mixing with cold ions, typically associated with a steep temperature gradient, to enhance the predicted intensity. Laming \& Feldman (1992) elaborated on a similar idea involving high-energy electrons in a burst model to match observed intensities. An alternative view of the 304 formation is the photoionization-recombination picture, where coronal EUV incident radiation photoionizes He II leading to a recombination cascade ultimately ending up in a 304 photon (Zirin 1988). Both of these processes may be of importance. Andretta et al. (2003) showed that at least for the quiet Sun, there are not enough coronal EUV photons produced to account for all of the 304 emission. The line is often used for the study of filaments and prominences (Liewer et al. 2009; Bi et al. 2012; Labrosse \& McGlinchey 2012). Another recent example of its use is for the study of spicules (Murawski et al. 2011) and heating events associated with them (De Pontieu et al. 2011).

In this paper, we describe a series of one-dimensional radiation-hydrodynamics simulations similar to those of CS2002. On the basis of the results, we derive a simplified helium model atom suitable for treating nonequilibrium helium ionization in three-dimensional numerical models. In addition, we perform an initial investigation of nonequilibrium ionization effects on the 10830 and 304 spectral lines. In Section 2, we describe the code, simulation setup, and assumptions for the construction of the simplified model atom. In Section 3, we describe our results. Finally, in Section 4, we draw conclusions.

\section{METHOD}

We use the one-dimensional radiation-hydrodynamics code RADYN (Carlsson \& Stein 1992, 1995, 1997; CS2002), which solves the equations of mass, momentum, charge and energy conservation, as well as the rate equations, on an adaptive grid. The simulations include a detailed treatment of nonequilibrium excitation, ionization, and radiative transfer from the atomic species $\mathrm{H}, \mathrm{He}$, and $\mathrm{Ca}$. Other elements are also included, but their contribution to the ionization energy and background opacity is based on the LTE assumption and read from a table produced by the Uppsala opacity program (Gustafsson 1973). We devote special attention to the internal energy balance equation:

$$
e=\frac{3 k T}{2}\left(n_{\mathrm{e}}+\sum_{i, j} n_{i, j}\right)+\sum_{i, j} n_{i, j} \chi_{i, j}
$$

where $e, n_{\mathrm{e}}, n_{i, j}$, and $\chi_{i, j}$ are the internal energy, electron density, population density, and excitation/ionization energy corresponding to the $i$ th state of the $j$ th ion. The two terms on the right side represent the contributions from thermal energy and ionization/excitation energy.

Hydrogen and singly ionized calcium are modeled with sixlevel atoms, and helium is modeled with a 33-level atom. Each line is described with 31-101 frequency points, whereas 6-90 frequency points are used for the various continua. The simulations carried out in this study are very similar to the one carried out in CS2002 with the major difference being the very detailed helium model atom included here. For more technical information about the code, we refer to this paper and the references therein.

\subsection{Model Atoms}

The hydrogen and calcium model atoms are identical to the ones used in CS2002. The helium model atom is a reduced version of an atomic model extracted from HAOS-DIPER. ${ }^{2}$ This original model has 75 energy levels: the ground state of neutral helium plus 48 excited states (14 singlet and 34 triplet) up to $n=5$, the ground state of He II and 24 excited states up to $n=5$, and He III. The energies of the He I and He II states are from the National Institute of Standards and Technology ${ }^{3}$ database and Sugar \& Corliss (1979), respectively. The model atom has 311 transitions: 255 lines and 56 continua. Line oscillator strengths for neutral helium are from the National Institute of Standards and Technology, and for the transitions in He II from Parpia \& Johnson (1982). All photoionization crosssections are from the OPACITY project. ${ }^{4}$ The collisional rates of bound-bound neutral helium transitions are from Sawey \& Berrington (1993) and the neutral bound-free rates from excited states are modeled with Seaton's semi-empirical formula for neutrals (Allen 1973, Section 18). For He II the bound-bound collisional rates are taken from CHIANTI (Dere et al. 2009), and the bound-free collisional rates from excited states are from Burgess \& Chidichimo (1983). The collisional ionization rate from the ground states of both He I and He II is from Arnaud \& Rothenflug (1985).

We reduce the number of levels in the atom from 75 to 33 by merging the neutral helium singlet $n=4$ states into one representative state. Similarly, the neutral helium singlet $n=5$ states, triplet $n=4$ states and triplet $n=5$ states are merged into three representative states. Also, the He II $n=4$ and $n=5$ states are merged into two representative states. The merging of levels is done by the method described in Bard \& Carlsson (2008), i.e., energies of merged levels are weighted averages and transition probabilities are computed under the assumption that the original levels have similar energies and identical departure coefficients (ratio between population density in non-LTE and LTE).

\subsection{Boundary Conditions}

The boundary conditions are the same as those in CS2002. Both the upper and lower boundaries are transmitting. The lower boundary is located at a fixed geometrical depth, corresponding to $480 \mathrm{~km}$ below $\tau_{500}=1$ in the initial atmosphere. The incoming characteristics at the lower boundary are prescribed, and they result in waves that propagate up through the atmosphere. The upper boundary is located at $10 \mathrm{Mm}$ and has a fixed temperature of $10^{6} \mathrm{~K}$ representing a corona. The upper boundary is irradiated by a fixed EUV incident radiation field (see Figure 1)

\footnotetext{
2 The HAO Spectral Diagnostic Package for Emitted Radiation:

http://www.hao.ucar.edu/modeling/haos-diper.

3 www.nist.gov

4 http://cdsweb.u-strasbg.fr/topbase/TheOP.html
} 


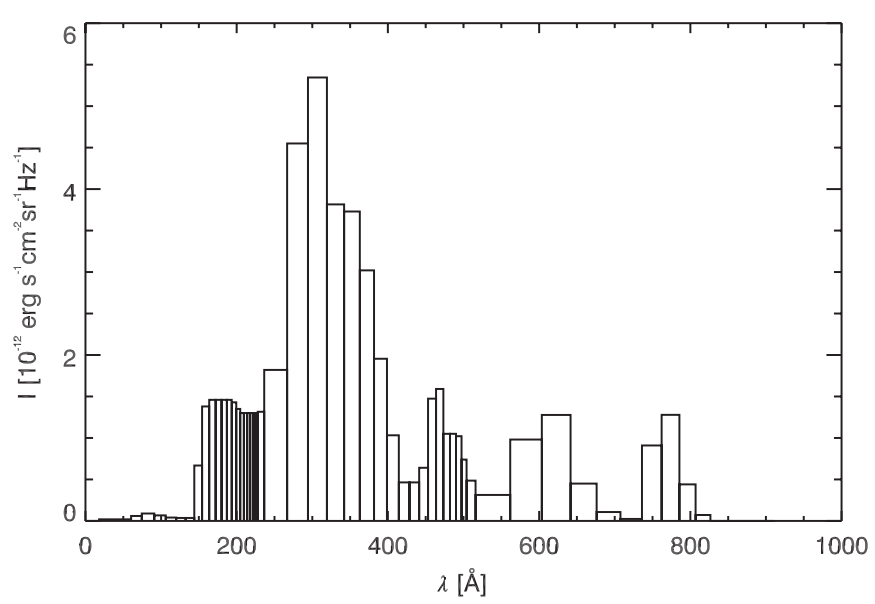

Figure 1. EUV incident radiation field at the upper boundary of the computational domain. The data have been binned at the same resolution as the employed frequency grid, while preserving the frequency-integrated energy flux.

that represents illumination by the corona. This radiation field is equal to that derived in Wahlstrom \& Carlsson (1994) on the basis of data from Tobiska (1991).

\subsection{Simulations}

We carried out three simulations, each running for $3600 \mathrm{~s}$ of solar time. The simulations differ only in the treatment of helium. We used the following setups: the 33-level model atom with nonequilibrium population densities (referred to as the NE-run), the 33-level atom with SE population densities (referred to as the SE-run), and a 3-level atom (derived in Section 2.5) with nonequilibrium population densities (referred to as the NE3-run).

Several smaller simulations are carried out in addition to the three main runs in order to determine the relaxation timescales of the helium ionization/recombination processes. The initial atmospheres of these runs are snapshots very similar to those found in the NE-run (as they are from a simulation where a 9-level He model atom was used instead of the 33-level model atom). These initial atmospheres contain the SE solution for the thermodynamic state of the atmosphere. The temperature is then increased by $1 \%$, and the populations are allowed to adjust, during which all other quantities are forced to remain constant with zero velocity. In addition, we computed the SE solution of the perturbed atmospheres.

\subsection{Initial Atmosphere}

The initial atmosphere for all runs is at rest, and all population densities are in SE. Figure 2 gives a comparison of the ion fractions, $f=n_{\text {ion }} / n_{\text {total }}$, and the corresponding LTE values. At low heights, helium is mostly neutral, and only from the upper chromosphere and upwards $(z>1.2 \mathrm{Mm})$ do we find He II fractions above $10^{-3}$. The He II fraction peaks somewhere in the transition region, above which all helium is effectively in the form of He III. LTE is a decent approximation in the photosphere, but it fails to reproduce realistic ion fractions from the chromosphere and up given that LTE does not take into account radiative transitions, whose most important contribution is the photoionization caused by coronal EUV radiation (Zirin 1975).

Because we suspect that the ionization state of helium affects the energy balance of the atmospheric gas, we carry out a rough numeric test of the temperature's sensitivity to the ionization
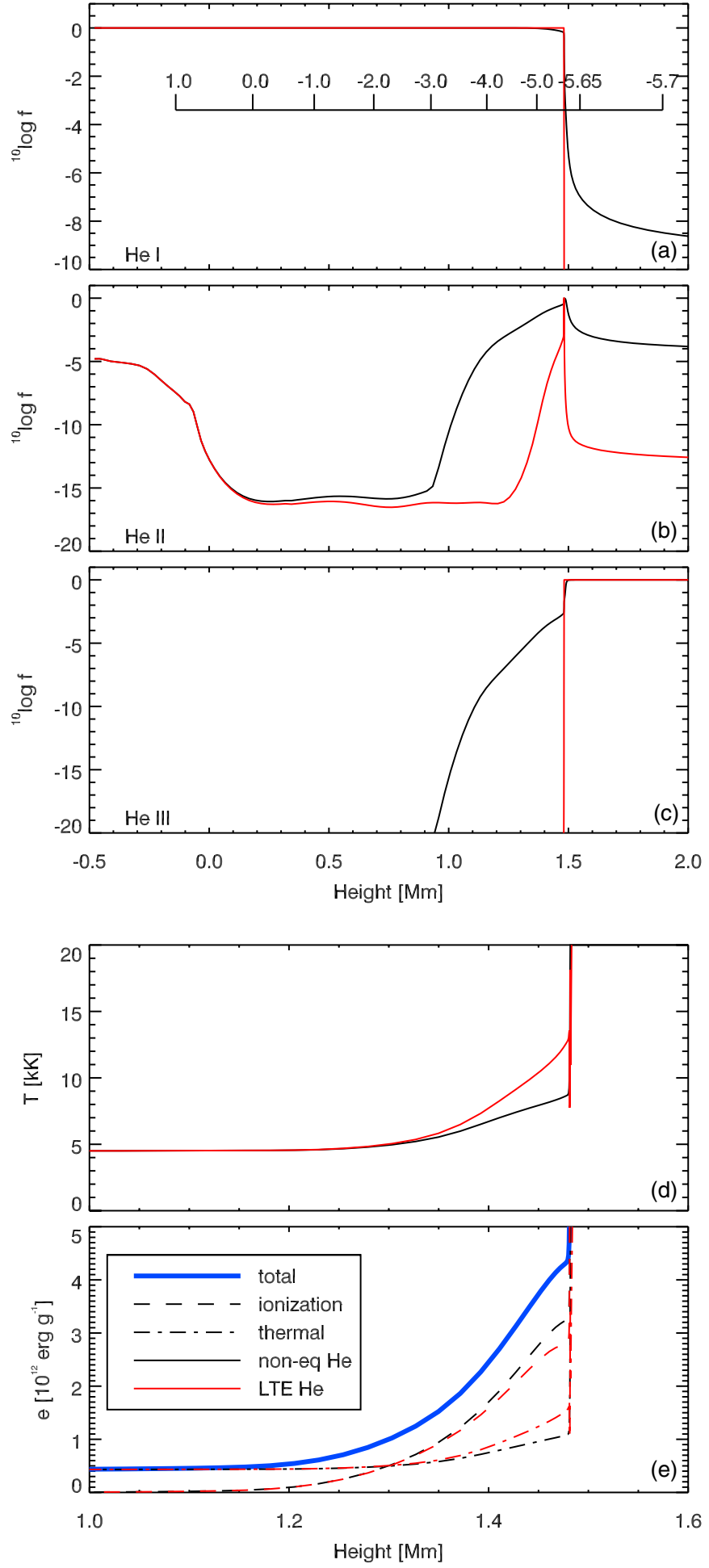

Figure 2. Panels (a)-(c): helium SE (black) and LTE (red) ion fractions of the initial atmosphere. The ${ }^{10} \log$ of the column mass $\left[\mathrm{g} \mathrm{cm}^{-2}\right]$ is indicated in the upper panel. Panel (d): comparison of the temperatures assuming SE (black) and LTE (red) helium ionization in the energy equation (Equation (1)). Panel (e): the terms of Equation (1) assuming SE (black) and LTE (red). The temperature is sensitive to the levels of helium ionization.

(A color version of this figure is available in the online journal.)

state of helium. This is done in the following way: we fix the internal energy of the initial atmosphere, set the helium population densities to their LTE value, and then re-solve the internal energy equation (Equation (1)) together with the charge 


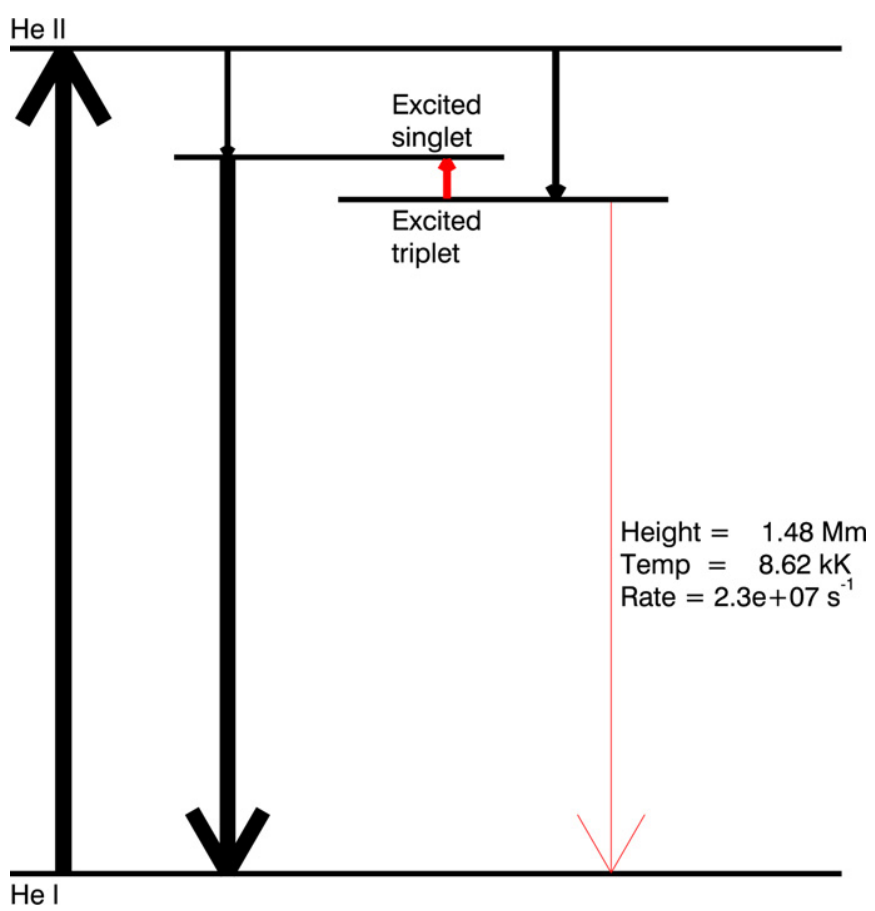

Figure 3. Net transition rates in the He I-He II system of the initial atmosphere. The red and black arrows represent collisional and radiative transitions, respectively. Their thickness is proportional to their absolute value; the maximum net rate is denoted in the figure as Rate. The driver of the system is the photoionization from the ground state of neutral helium, which is balanced mainly by radiative recombination cascades. This picture is qualitatively valid from the temperature minimum at a height of $0.9 \mathrm{Mm}$ to the transition region at $1.5 \mathrm{Mm}$.

(A color version of this figure is available in the online journal.)

conservation equation (as ionized helium is a significant source of electrons).

We compare the energy balance between thermal energy and ionization energy in the SE and LTE cases in the two lower panels of Figure 2 (note the different height scale). Moving upwards from the photosphere, the temperature in the SE and LTE cases starts to deviate in the upper chromosphere. The density of He II ions is several orders of magnitude higher in SE than compared with what is predicted by LTE. This leads to a higher ionization energy, which, because the total energy is fixed, must result in a decrease of the thermal energy. Continuing into the transition region, the LTE temperature drops abruptly before it rises to the coronal value. The sudden drop happens because helium in LTE goes from neutral to fully ionized in a very short temperature interval centered at about $20 \mathrm{kK}$. This leads to an increase in the ionization energy and a subsequent fall in the thermal energy that is not present with helium in SE. This illustrates that the temperature in the solar chromosphere and transition region is sensitive to the helium ionization balance.

The ionization state in the upper chromosphere of the initial model atmosphere is set mostly by photoionization from the ground states of $\mathrm{He}$ I and He II followed by a radiative recombination cascade through the excited states back to the ground states. This is illustrated in Figures 3 and 4.

\subsection{Simplified Model Atom}

Figures 3 and 4 do not show how important the photoionization from the excited states is, only that it is less frequent than radiative recombination. We make the simplifying assumption that the excited states act solely as intermediate steps in recombination cascades and that they do not serve as states from which

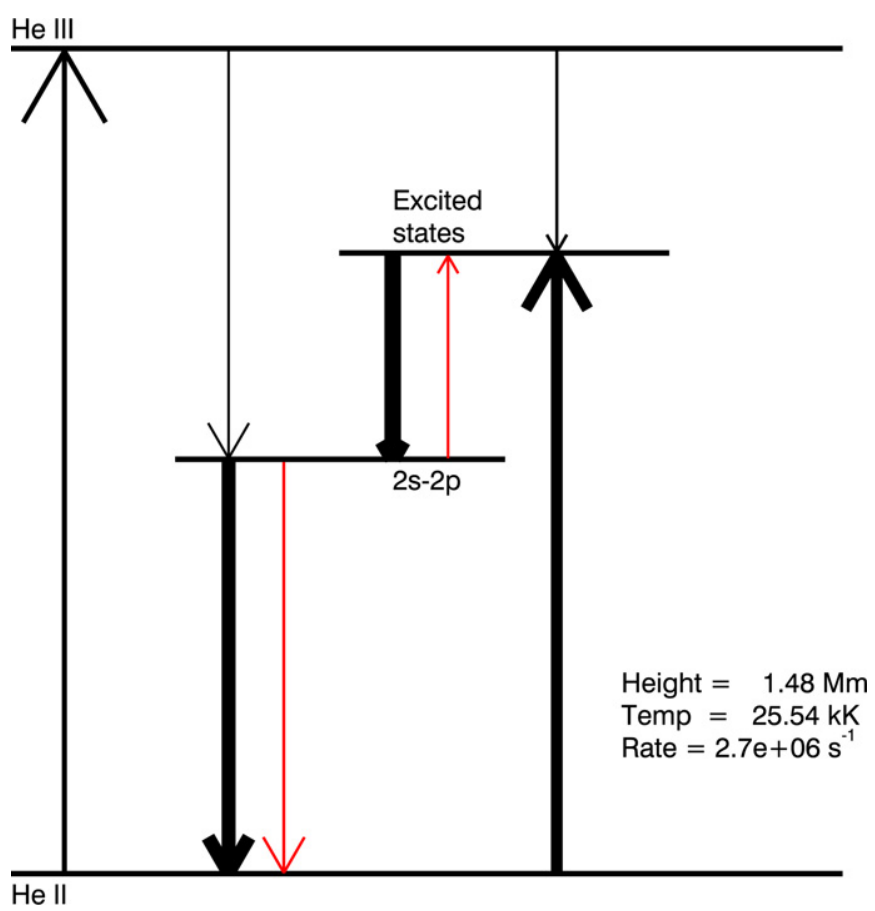

Figure 4. Net transition rates in the He II-He III system at the base of the transition region in the same format as Figure 3. Similar to the $\mathrm{He} \mathrm{I}-\mathrm{He}$ II system, photoionization from the ground state, balanced by radiative recombination cascades, dominate in setting the ionization degree. At higher temperatures, collisional ionization becomes more important.

(A color version of this figure is available in the online journal.)

photoionization is taking place. On the basis of this assumption, we set up a model atom with only three levels: ground-state He I ground-state $\mathrm{He}$ II and He III.

In addition to the ordinary collisional and radiative rates between these levels, we add an extra recombination rate for each of the two ions, He II and He III. This extra rate is meant to model the net recombination to excited states.

The number of photoionizations $\left(n_{i} R_{i c}\right)$, spontaneous recombinations $\left(n_{c} R_{c i}^{\mathrm{sp}}\right)$ and induced recombinations $\left(n_{c} R_{c i}^{\mathrm{in}}\right)$ between a level $i$ and its overlying continuum level $c$ can be expressed as follows:

$$
\begin{gathered}
n_{i} R_{i c}=n_{i} f_{i c}\left(J_{v}\right) \\
n_{c} R_{c i}^{\mathrm{in}}=n_{c} n_{\mathrm{e}} f_{c i}^{\mathrm{in}}\left(T, J_{v}\right) \\
n_{c} R_{c i}^{\mathrm{sp}}=n_{c} n_{\mathrm{e}} f_{c i}^{\mathrm{sp}}(T),
\end{gathered}
$$

where $n_{i}$ and $n_{c}$ are the population densities of the $i$ th and $c$ th level and $J_{v}$ is the (frequency-dependent) mean intensity. The functions $f_{i c}\left(J_{v}\right), f_{c i}^{\text {in }}\left(T, J_{v}\right)$ and $f_{c i}^{\mathrm{sp}}(T)$ depend on temperature and mean intensity (Mihalas 1978). The net recombination from the $c$ th ionization stage to excited states of the $(c-1)$ th ionization stage, $P_{c}$, can now be expressed as follows:

$$
P_{c}=n_{c} n_{\mathrm{e}} \sum_{i}\left(f_{c i}^{\mathrm{sp}}+f_{c i}^{\mathrm{in}}\right)-\sum_{i} n_{i} f_{i c},
$$

where $i$ in the sum represents the excited states.

We want to model this with an effective recombination rate $P_{c}=n_{c} n_{\mathrm{e}} r_{c}$, where $r_{c}$ is dependent on temperature only. This leads to the following expression for $r_{c}$ :

$$
r_{c}=\sum_{i}\left(f_{c i}^{\mathrm{sp}}(T)+f_{c i}^{\mathrm{in}}\left(T, J_{\nu}\right)\right)-\frac{\sum_{i} n_{i} f_{i c}\left(J_{v}\right)}{n_{c} n_{\mathrm{e}}} .
$$




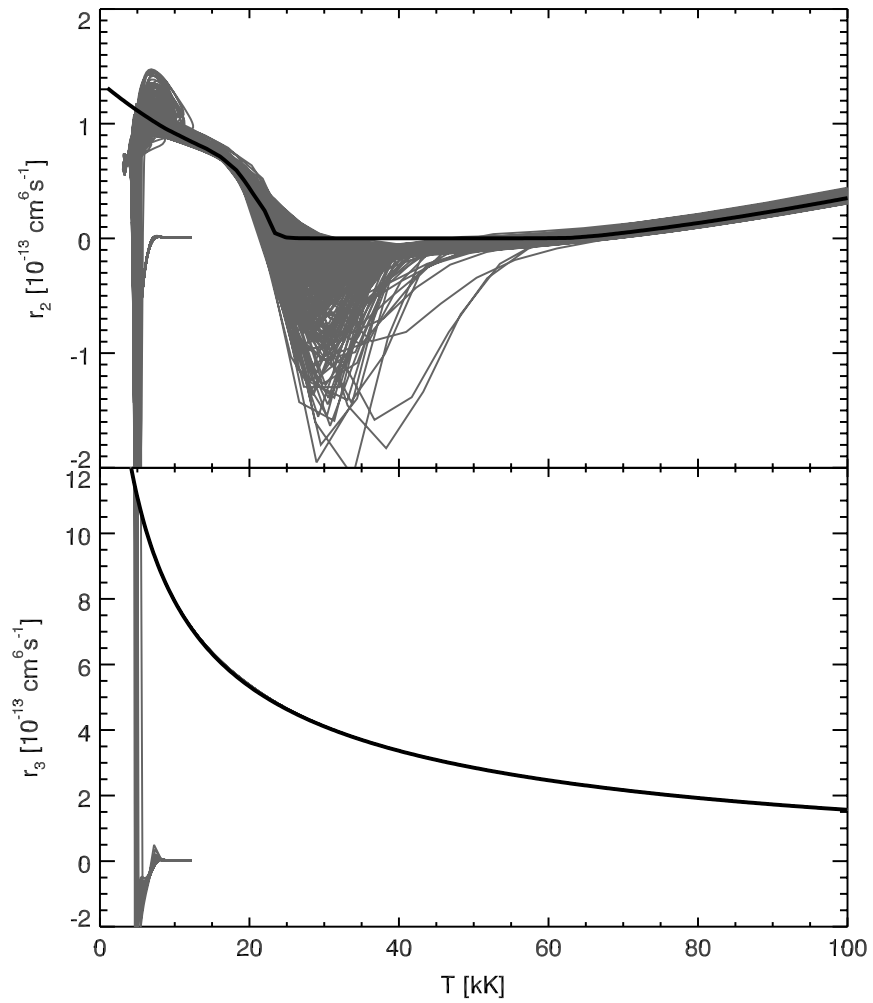

Figure 5. Effective recombination rate coefficient, $r_{c}$, as a function of temperature for neutral (upper panel) and singly ionized helium (lower panel). The gray lines correspond to different snapshots from the NE-run simulation and the black line represents the value chosen for the model atom. Negative values correspond to net photoionization from excited states.

If photoionization from excited states and induced recombination are negligible, $r_{c}$ will be dominated by spontaneous recombination that is dependent on temperature only.

In Figure 5, we show $r_{c}$ for all snapshots of the NE-run. The upper panel shows recombination from $\mathrm{He}$ II to $\mathrm{He}$ I. At the low temperature end $(T=5 \mathrm{kK})$, there is a steep downturn and a horizontal extension toward higher temperatures at $r_{2}=0$. These are points in the photosphere. Between $T=20 \mathrm{kK}$ and $T=60 \mathrm{kK}$, there is net photoionization (negative $r_{2}$ ). We choose to model $r_{2}$ with its time average, except at the low temperature end, where we used an increase in recombination with decreasing temperature. We neglect the net photoionization between $T=20 \mathrm{kK}$ and $T=60 \mathrm{kK}$, as there is very little neutral helium at those temperatures; the error we make is thus small. Similarly, we ignore the downturn at the low temperature end. These points correspond to the photosphere where there is little He II and the high density there causes the rates to be dominated by collisional processes.

The lower panel of Figure 5 shows $r_{3}$. There is very little spread, except in the low temperature end, which confirms the radiative recombination cascade scenario illustrated in Figure 4. We chose the time-averaged values of $r_{3}$ for the three-level model atom, with an extrapolation at the low temperature end.

\section{RESULTS}

\subsection{Time-dependent Ionization with the Full Model Atom}

As waves propagate upwards in the atmosphere, the thermodynamic conditions change too fast for the ion densities to adjust, resulting in nonequilibrium ion fractions. Details of the ion fractions and net transition rates from two snapshots of the NE-run are shown in Figure 6. The simulations reveal, as far as the helium transitions are concerned, two types of situations: preshock and postshock, which are represented in the two chosen snapshots. In the chromospheric preshock phase $(t=2930 \mathrm{~s}, z=[-0.08,-0.02] \mathrm{Mm}$, where $z=0 \mathrm{Mm}$ is defined to be where the temperature is $100 \mathrm{kK}$ ), there is a net ionization. This is driven by the EUV incident radiation, which photoionizes neutral helium. Counteracting radiative recombination to excited states is taking place, but the rates are not large enough to balance the photoionization. When the shock passes and compresses the gas, the electron density increases by both hydrogen ionizing and the compression itself (CS2002). The radiative recombination rate coefficient depends linearly on electron density, whereas the photoionization rate coefficient is independent of electron density (Equations (2)-(4)). This results in an increase of the net recombination in the postshock phase $(z<-0.08 \mathrm{Mm})$. Collisions play only a minor role in the chromosphere. In the snapshot $t=2950 \mathrm{~s}$, the postshock phase is slowly adjusting itself back to the preshock phase where photoionization dominates, concurrent with the depletion of electrons which is due to the recombination of hydrogen.

The effects of He III are most important in the transition region where $T \sim 10-100 \mathrm{kK}$, given that the relative amount of neutral hydrogen and helium here is small. Where $T<50 \mathrm{kK}$, He II is behaving similarly to He I: photoionizations from the ground state of He II, driven by the EUV incident radiation, is roughly balanced by radiative recombinations to the excited states. At $T>50 \mathrm{kK}$, however, collisional ionization from the ground state of He II replaces photoionization as the dominating rate, peaking at $T=90 \mathrm{kK}$. Also, note the nonnegligible collisional ionization from the metastable He II $2 s$ state. In the transition region preshock phase $(t=2930 \mathrm{~s})$, the radiative recombination dominates, causing a net recombination. When the shock passes, the temperature rises as a result of the compression, and the higher temperature results in net ionization as the net collisional ionization rates grow large $(t=2950 \mathrm{~s})$. When the plasma cools down, the net rates adjust themselves back toward the preshock phase.

\subsubsection{Time-dependent Ionization with the Simplified Model Atom}

Ionization fractions obtained with the simplified model atom (from the NE3-run) are shown together with the NE-run ion fractions in Figure 6. We have replaced 30 atomic states in the simplified model atom with 2 extra recombination rates. Processes involving the removed states are not taken into account, and this leads to a small shift in the position of the transition region compared with the NE-run. We have set $z=0 \mathrm{Mm}$, where $T=100 \mathrm{kK}$ in both simulations, to be able to compare the structure of the transition region directly from the figure.

The simplified model atom reproduces the ion fraction from the NE-run very well, despite the neglected effects. Processes not taken into account include three-body recombination to and ionization from the excited states of both He I and He II. Figure 6 shows that these effects are subordinate.

\subsection{Relaxation Timescales}

We define a relaxation timescale in the same way as in CS2002. As described in Section 2.3, we have initial $\left(n_{0}\right)$, time-dependent $(n(t))$ and final $\left(n_{\infty}\right)$ population densities from representative snapshots where the temperature is perturbed. The relaxation timescales, $\tau_{R}$, are obtained numerically by 

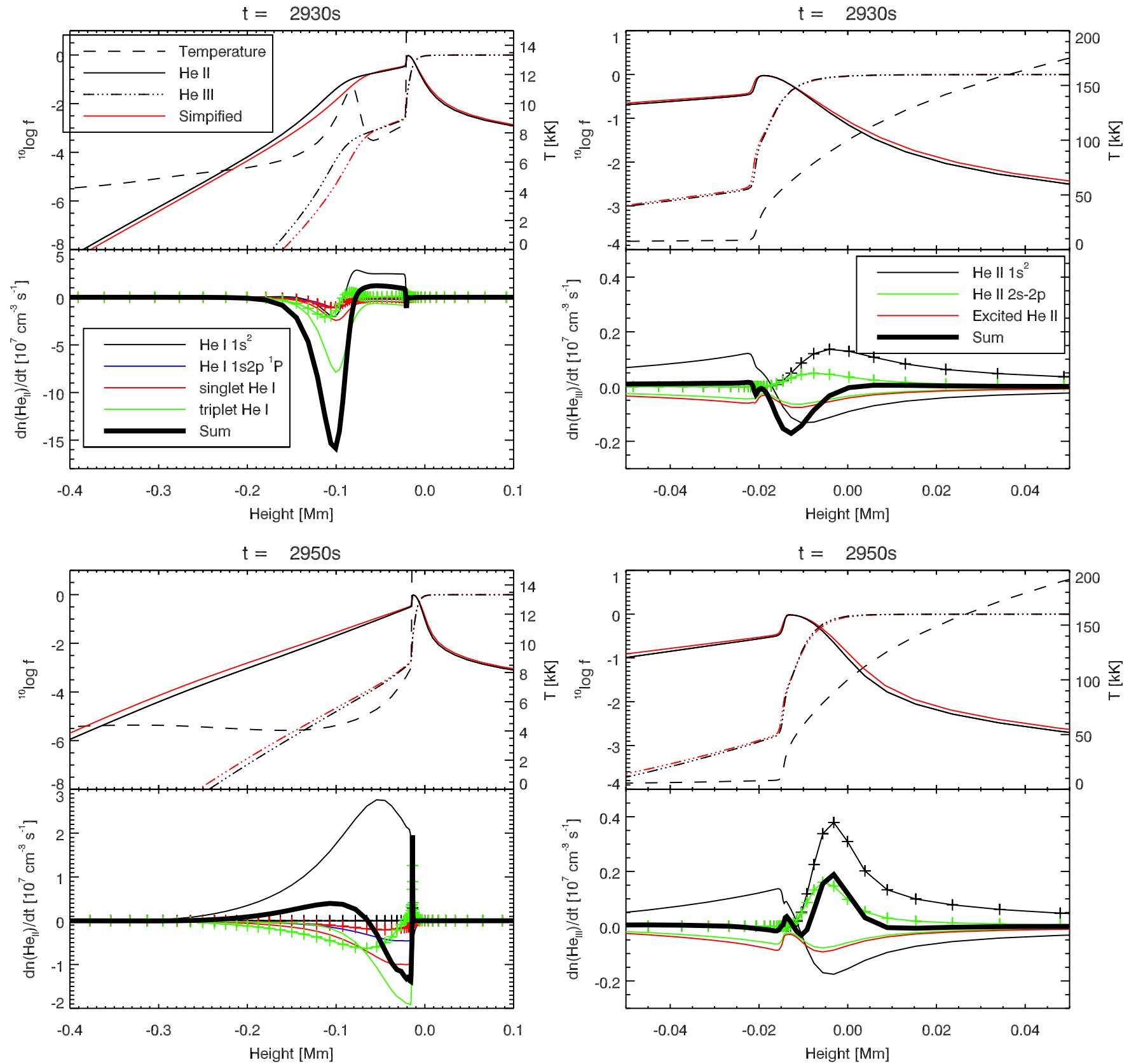

Figure 6. Ion fractions and net rates for two representative NE-run snapshots. Ion fractions from NE3-run are shown for comparison. The lines with plus signs represent collisional processes. Each row corresponds to a snapshot. The left column shows the conditions in the chromosphere and rates into the ground state of He II. The right column shows the conditions in the transition region and the rates into He III. To be able to better compare the structure of the transition region in the two runs, we have set $z=0 \mathrm{Mm}$ at $T=100 \mathrm{kK}$.

(A color version of this figure is available in the online journal.)

fitting the time-dependent population density of the ground state of He II to the general solution of a rate equation, assuming two levels and constant transition rate coefficients:

$$
n(t)=n_{\infty}-\left(n_{\infty}-n_{0}\right) e^{-t / \tau_{R}} .
$$

Figure 7 shows the relaxation timescales of the three representative snapshots featuring both the preshock phase and the postshock phase. In the corona and photosphere, large rates result in short relaxation timescales. The relaxation timescale is largest around the photosphere/chromosphere interface at a column mass of ${ }^{10} \log m_{c} \approx-3 \mathrm{~g} \mathrm{~cm}^{-2}$ with typically a few hundreds of seconds. In the chromosphere, it ranges from a couple of tens and up to about a hundred seconds.
A wave front is building up at ${ }^{10} \log m_{c}=-2.7 \mathrm{~g} \mathrm{~cm}^{-2}$ in the red atmosphere. At this stage, a shock has just passed through the transition region and the chromospheric relaxation timescale is adjusting itself back to the preshock values of about 60-100 s. The shock propagates through the chromosphere (blue line) and reduces the timescale to its postshock value of a few tens of seconds (green line).

In the transition region at ${ }^{10} \log m_{c}=-5.6 \mathrm{~g} \mathrm{~cm}^{-2}$, there is a sharp spike in $\tau_{R}$, marking the position of where the ionization shifts from a balance between $\mathrm{He}_{\mathrm{I}}$ and $\mathrm{He}_{\mathrm{II}}$ to a balance between He II and He III. The definition of $\tau_{R}$ is based on a two-level system and will not be very meaningful when three levels are important at the same time. However, the spike itself 

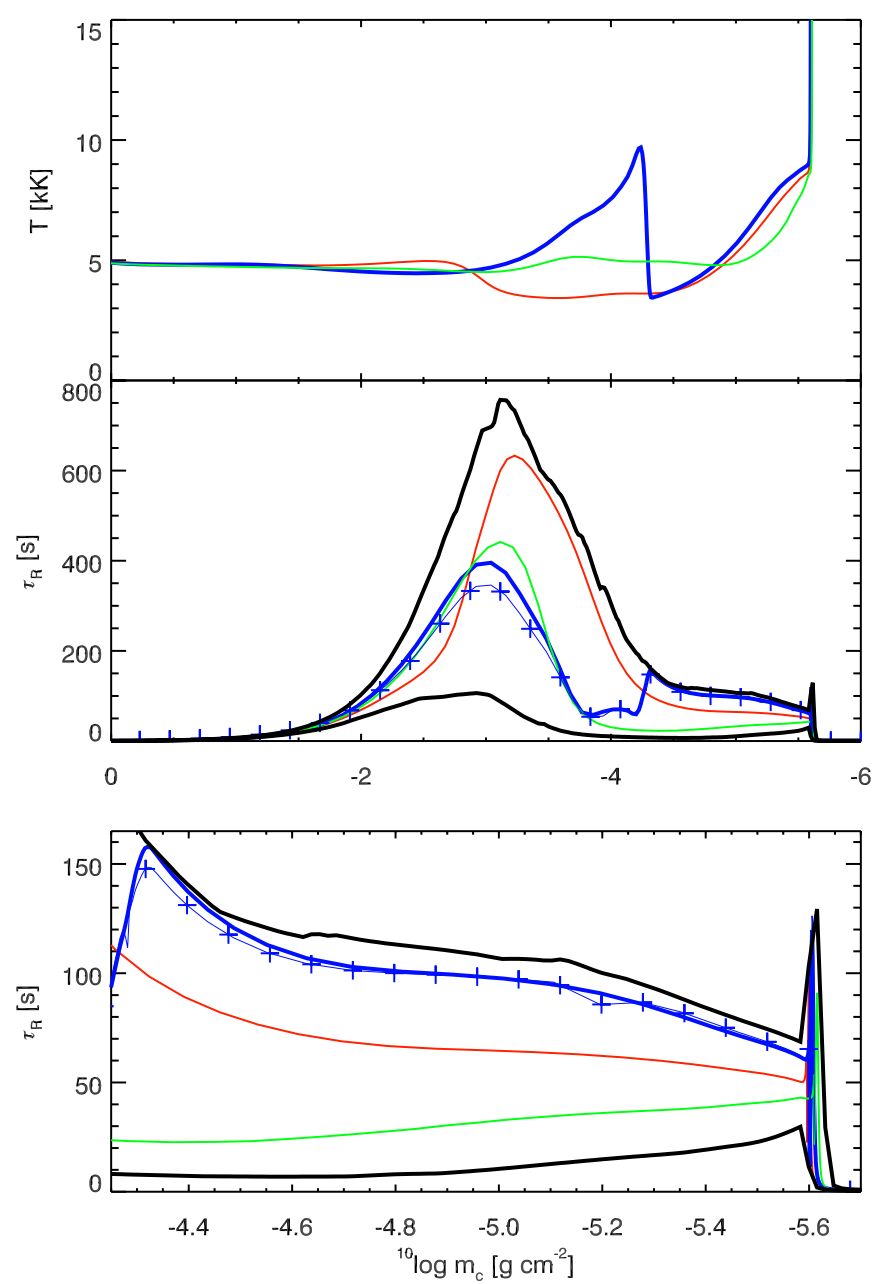

Figure 7. Temperature profiles of three snapshots (upper panel) and their corresponding relaxation timescales (middle and lower panel). The middle panel shows the whole computational domain, the lower panel is a blowup of the chromosphere and transition region. Each snapshot is shown in its own color (red, green, and blue) in all panels. The maximum and minimum timescales obtained are shown in thick black. The timescale obtained with the simplified model atom is show for one atmosphere only (thin, blue curve with plus signs).

(A color version of this figure is available in the online journal.)

is within the region where the balancing is between He II and He III, i.e., Equation (7) is a decent approximation and provides a meaningful estimate.

The simplified model atom reproduces the relaxation timescales we get with the full 33-level atom very well. The model atom includes the most important transition processes-clearly, these are also dominant in setting the timescales of the system.

\subsubsection{Timescales of Various Processes}

We assume constant transition rates and use the analysis tools developed in Judge (2005). The set of rate equations in a static atmosphere constitutes a set of first-order differential equations that can be expressed as a matrix equation:

$$
\dot{\mathbf{n}}=P \mathbf{n},
$$

with the solution

$$
\mathbf{n}=\sum_{i} c_{i} \mathbf{v}_{i} e^{\lambda_{i} t}
$$

where $P$ is the rate matrix, $\mathbf{n}$ is a vector containing the population densities, $\mathbf{v}_{i}$ and $\lambda_{i}$ are the corresponding eigenvectors and eigenvalues of the rate matrix, and the coefficients, $c_{i}$, are constants depending on the initial conditions. The eigenvectors represent different relaxation processes. One of the eigenvalues is zero, and the corresponding eigenvector is proportional to the SE solution. Each of the processes has a corresponding relaxation timescale, given by $\tau_{i}=-1 / \lambda_{i}$. The smallest nonzero eigenvalue (in absolute value because they are all negative) corresponds to the slowest relaxation process and thereby determines the relaxation timescale of the system as a whole.

By inspecting the eigenvectors in the 33-level model atom, we find that the balancing between the ground states of $\mathrm{He}$ I and He II is the slowest process in the chromosphere. The second slowest, by a factor of five to six, is the balancing between the ground state of He II and He III. This is valid in both the pre- and postshock phases. In the transition region where $T=60 \mathrm{kK}$ (corresponding to the peak in the relaxation timescale, see Figure 7), balancing between the ground state of He II and He III is the slowest process. All other processes happen on timescales less than a second.

\subsection{Nonequilibrium Effects}

The relaxation timescales of helium in the chromosphere and transition region ranges from a few tens of seconds to about one hundred seconds. When shock waves propagate through these regions, the hydrodynamic conditions may change too fast for the ionization balance to adjust. SE is an often-used assumption when producing synthetic spectra, but this may not be a good assumption for the spectral lines of helium if there are rapid changes in the solar atmosphere. To obtain a feel for the types of errors such an assumption would cause, we perform an initial investigation of the nonequilibrium effects of He I 10830 and He II 304.

\subsubsection{He II 304}

The resonance line He II 304 has two components, $1 s^{2} S_{1 / 2}^{\mathrm{e}}-2 p^{2} P_{1 / 2,3 / 2}^{\mathrm{o}}$ (excluding the forbidden $1 s^{2} S_{1 / 2}^{\mathrm{e}}-2 s^{2} S_{1 / 2}^{\mathrm{e}}$ ), treated separately in our simulations. We investigated the component with the largest oscillator strength $\left(1 s^{2} S_{1 / 2}^{\mathrm{e}}-2 p^{2} P_{3 / 2}^{\mathrm{o}}\right)$.

Figure 8 compares the formation of the 304 line in the NE-run and the SE-run. A shockwave-induced sawtooth pattern is present in both runs, and the intensity of the line generally increases as the shock front passes the transition region where the line forms.

We first discuss the NE line formation. During the shock front passage, the temperature increases on a timescale shorter than the ionization-recombination timescale (see Section 3.2), and the He II is therefore not ionized away. The compression work done by the shock is converted mainly into thermal energy and not to ionization energy, and the temperature increases strongly.

Investigation of the rates show that the line photons are produced mainly by collisional excitation from the ground state. The large temperature rise leads to increased collisional excitation and thus strong emission in the 304 line as the shock passes the transition region. This is why the line core intensity and temperature at the formation height are so strongly correlated.

In contrast, in the SE-run, He II is instantaneously ionized as the shock front passes. This has two consequences, both of 


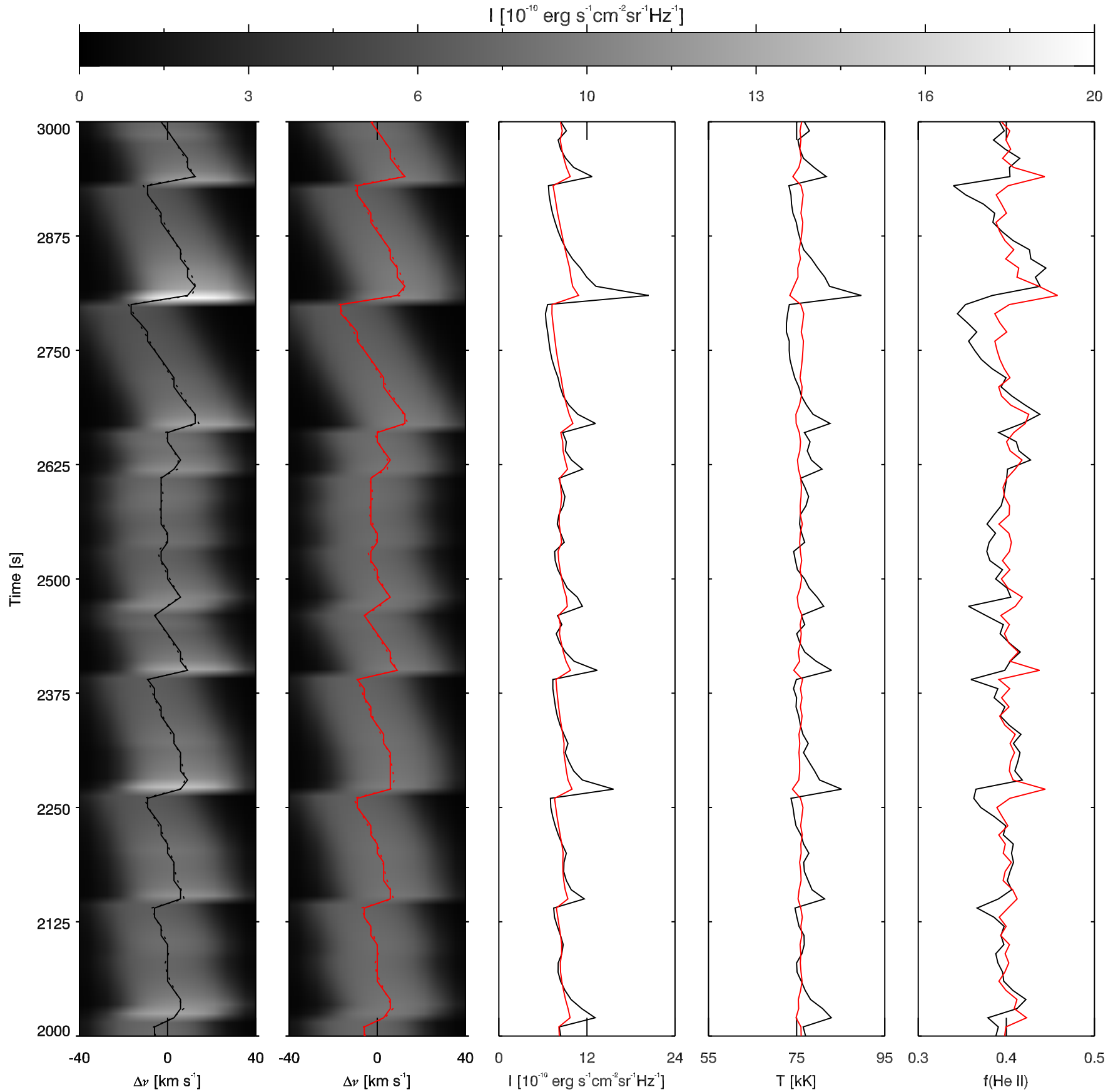

Figure 8. Formation of the He II 304 line. From left to right: emergent intensity in the NE-run, emergent intensity in the SE-run, line core intensities, temperature at line core optical depth unity and fraction of He II at line core optical depth unity $(\tau=1)$. In the three rightmost panels, the NE-run values are black and SE-run values are red. The line core is defined as the wavelength where the emergent intensity is at its maximum and indicated by the solid lines in the two leftmost panels. Also indicated in these panels is the plasma velocity at $\tau=1$ (dotted lines), which overlaps almost completely with the Doppler shift of the line core.

(A color version of this figure is available in the online journal.)

which reduce the number of 304 photons produced. First, the temperature increase is smaller given that the increase of energy goes into ionizing helium. Second, the instantaneous ionization to He III lowers the 304 opacity and shifts the $\tau=1$ height down, where the temperature is lower. The line intensity in the SE-run is thus lower than in the NE-run. A good example of this can be seen at $t=2810 \mathrm{~s}$.

The plasma velocity at the formation height coincides with the Doppler shift of the line. This suggests that the line is formed in a thin atmospheric layer.

\subsubsection{He I 10830}

The He I 10830 line forms when continuum photons from the photosphere are scattered and absorbed in the chromosphere by neutral helium atoms residing in the $2 s^{3} S_{1}^{\mathrm{e}}$ state in the triplet system of He I. This is the lower level of the three 10830 transitions, $2 s^{3} S_{1}^{\mathrm{e}}-2 p^{3} P_{0,1,2}^{\mathrm{o}}$.

The triplet states are populated through recombination of He II (Avrett et al. 1994; Mauas et al. 2005). This recombination happens on very short timescales compared with the timescales 

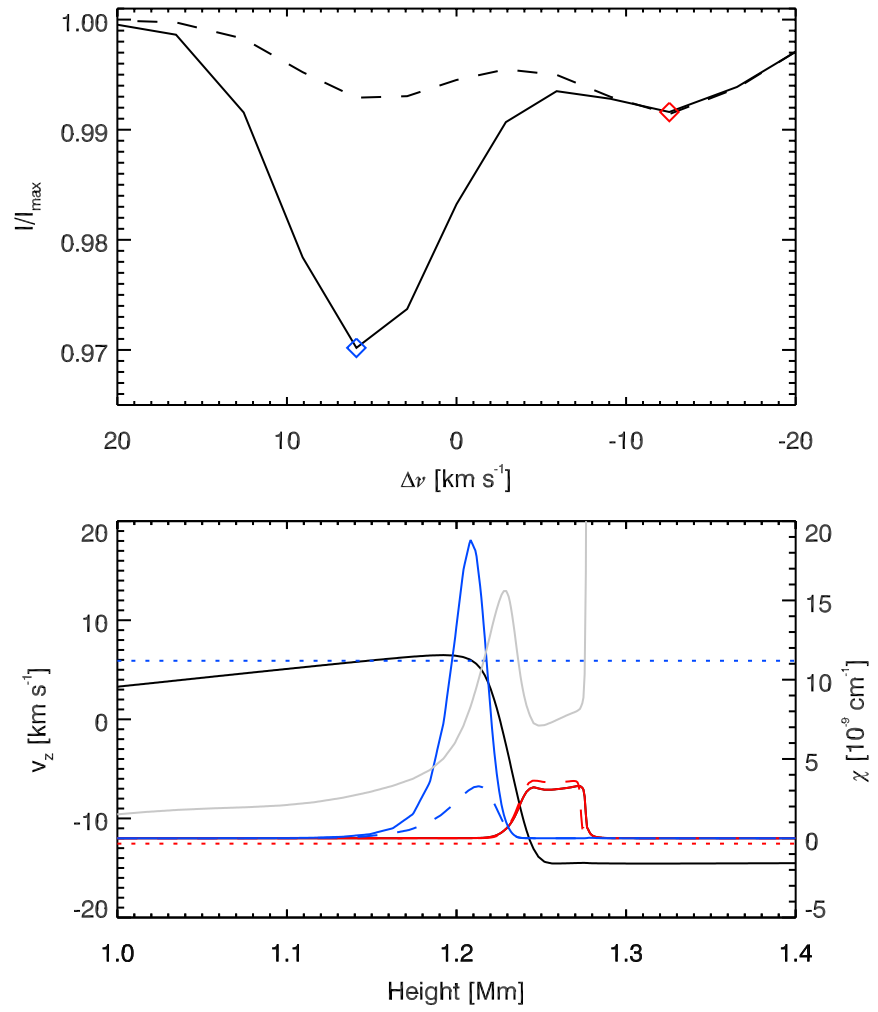

Figure 9. Formation of the He I 10830 line profile at $t=1650 \mathrm{~s}$. Upper panel: emergent intensity for the NE-run (solid) and SE-run (dashed). The red and blue diamonds indicate the wavelengths used in the lower panel. Lower panel: velocity in the atmosphere (solid black, scale to the left) and opacity at the two selected wavelengths (blue and red solid and dashed, scale to the right). The blue curves correspond to the frequency of the blue diamond in the upper panel (solid for NE, dashed for SE), the red curves for the frequency of the red diamond. The red and blue frequencies are also indicated on the velocity scale by the horizontal dotted lines in the lower panel. In addition, we show the temperature in gray. By coincidence, the line profiles resemble the 10830 blend, so we stress that the profiles shown here are only the strongest component of the line. The red dip in the line is formed in the preshock phase where matter is falling downward, and the blue dip is formed in the shock wave.

(A color version of this figure is available in the online journal.)

of the ionization (see Section 3.2). This means that the $2 s^{3} S_{1}^{\mathrm{e}}$ population density, and hence the opacity, is always adjusted to the amount of He II. Our simulations treat each of the three components of the 10830 line separately, and we choose to investigate the component with the largest oscillator strength $\left(2 s^{3} S_{1}^{\mathrm{e}}-2 p^{3} P_{2}^{\mathrm{o}}\right)$.

The upper panel of Figure 9 shows the line profiles for both $\mathrm{NE}$ and SE runs at $t=1650 \mathrm{~s}$. The line profile has two depression cores at positive and negative Doppler shifts relative to the rest wavelength. The NE profile has a much deeper blueshifted depression than does the SE profile.

The lower panel shows the opacity at the wavelengths of the maximum depression together with the structure of the atmosphere. The red-shifted absorption is formed in the downflowing preshock material, and the blue-shifted component is formed in the upflowing material behind the shock front. The Doppler shift of the minima of the absorption components is nearly equal to the gas velocity in the line-forming region.

The opacity of the blue-shifted component in the SE-run is much lower than in the NE-run, explaining the depth difference of the depression cores. In this region, ionization equilibration timescales are around $50 \mathrm{~s}$ (see Figure 7). The helium population densities of the SE-run equilibrates instantaneously to the increased electron density associated with the shock, resulting in a smaller amount of He II. Because He II is acting as a reservoir for the triplet states, the 10830 opacity decreases accordingly.

\section{CONCLUSIONS}

We have carried out several one-dimensional radiationhydrodynamic simulations in order to study the processes determining helium ionization and the timescales at which they work, and we have performed an initial investigation of the formation of the He I 10830 and He II 304 lines.

The helium ionization state is far from in LTE in the upper chromosphere and transition region. Photoionization and collisional ionization from the ground states are the determining processes in setting the level of helium ionization. These processes work on timescales of up to $100 \mathrm{~s}$. Thermodynamic conditions are sensitive to the ionization fraction of helium. Using an equation of state with helium ionization assumed to be in LTE in numerical models will therefore give erroneous results. To rectify this, we have constructed a simplified threelevel helium model atom, based on the driving mechanisms, that reproduces the nonequilibrium ionization fractions quite well. The rate equations of the simplified model atom are simple enough for inclusion in multidimensional stellar atmosphere codes. We plan to include nonequilibrium ionization of helium in the three-dimensional radiation-magnetohydrodynamics code Bifrost.

We have shown that the formation of He I 10830 and He II 304 lines is sensitive to nonequilibrium effects. Both lines show behavior that is not reproduced when SE is assumed. We therefore recommend exercising caution when observations are interpreted on the basis of SE computations.

This research was supported by the Research Council of Norway through the grant "Solar Atmospheric Modelling" and by the European Research Council under the European Union's Seventh Framework Programme (FP7/2007-2013)/ ERC grant agreement No. 291058. We thank the referee for helpful comments in the preparation of this manuscript.

\section{REFERENCES}

Allen, C. W. 1973, Astrophysical Quantities (3rd ed.; London: Athlone) Andretta, V., Del Zanna, G., \& Jordan, S. D. 2003, A\&A, 400, 737

Arnaud, M., \& Rothenflug, R. 1985, A\&AS, 60, 425

Avrett, E. H., Fontenla, J. M., \& Loeser, R. 1994, in IAU Symp. 154, Infrared Solar Physics, ed. D. M. Rabin, J. T. Jefferies, \& C. Lindsey (Cambridge: Cambridge Univ. Press), 35

Bard, S., \& Carlsson, M. 2008, ApJ, 682, 1376

Bi, Y., Jiang, Y., Li, H., Hong, J., \& Zheng, R. 2012, ApJ, 758, 42

Burgess, A., \& Chidichimo, M. C. 1983, MNRAS, 203, 1269

Cao, W., Goode, P. R., Ahn, K., et al. 2012, in ASP Conf. Ser. 463 Second ATST-EAST Meeting: Magnetic Fields from the Photosphere to the Corona., ed. T. R. Rimmele, A. Tritschler, F. Wöger, M. Collados Vera, H. Socas-Navarro, R. Schlichenmaier, M. Carlsson, T. Berger, A. Cadavid, P. R. Gilbert, P. R. Goode, \& M. Knölker (San Francisco, CA: ASP), 291 Carlsson, M., \& Stein, R. F. 1992, ApJL, 397, L59

Carlsson, M., \& Stein, R. F. 1995, ApJL, 440, L29

Carlsson, M., \& Stein, R. F. 1997, ApJ, 481, 500

Carlsson, M., \& Stein, R. F. 2002, ApJ, 572, 626

Centeno, R., Trujillo Bueno, J., Uitenbroek, H., \& Collados, M. 2008, ApJ, 677,742

Collados, M., Lagg, A., Díaz Garcí A, J. J., et al. 2007, in ASP Conf. Ser. 368, The Physics of Chromospheric Plasmas, ed. P. Heinzel, I. Dorotovič, \& R. J. Rutten (San Francisco, CA: ASP), 611

De Pontieu, B., McIntosh, S. W., Carlsson, M., et al. 2011, Sci, 331, 55

Dere, K. P., Landi, E., Young, P. R., et al. 2009, A\&A, 498, 915

Gudiksen, B. V., Carlsson, M., Hansteen, V. H., et al. 2011, A\&A, 531, A154

Gustafsson, B. 1973, UppAn, 5, 1

Harvey, K. L., \& Recely, F. 2002, SoPh, 211, 31 
Howard, R. A., Moses, J. D., Vourlidas, A., et al. 2008, SSRv, 136, 67

Ji, H., Cao, W., \& Goode, P. R. 2012, ApJL, 750, L25

Jordan, C. 1975, MNRAS, 170, 429

Judge, P. G. 2005, JQSRT, 92, 479

Labrosse, N., \& McGlinchey, K. 2012, A\&A, 537, A100

Laming, J. M., \& Feldman, U. 1992, ApJ, 386, 364

Leenaarts, J., Carlsson, M., Hansteen, V., \& Gudiksen, B. V. 2011, A\&A, 530, A124

Leenaarts, J., Carlsson, M., Hansteen, V., \& Rutten, R. J. 2007, A\&A, 473, 625

Leenaarts, J., \& Wedemeyer-Böhm, S. 2006, A\&A, 460, 301

Lemen, J. R., Title, A. M., Akin, D. J., et al. 2012, SoPh, 275, 17

Liewer, P. C., de Jong, E. M., Hall, J. R., et al. 2009, SoPh, 256, 57

Mauas, P. J. D., Andretta, V., Falchi, A., et al. 2005, ApJ, 619, 604
Mihalas, D. 1978, Stellar Atmospheres (2nd ed.; San Francisco: Freeman)

Murawski, K., Srivastava, A. K., \& Zaqarashvili, T. V. 2011, A\&A, 535, A58

Parpia, F. A., \& Johnson, W. R. 1982, PhRvA, 26, 1142

Sawey, P. M. J., \& Berrington, K. A. 1993, ADNDT, 55, 81

Sheeley, N. R., Jr. 1980, SoPh, 65, 229

Sollum, E. 1999, Master's thesis, Univ. Oslo

Sugar, J., \& Corliss, C. 1979, JPCRD, 8, 865

Tobiska, W. K. 1991, JATP, 53, 1005

Wahlstrom, C., \& Carlsson, M. 1994, ApJ, 433, 417

Xu, Z., Lagg, A., Solanki, S., \& Liu, Y. 2012, ApJ, 749, 138

Zirin, H. 1975, ApJL, 199, L63

Zirin, H. 1988, Astrophysics of the Sun (Cambridge: Cambridge Univ. Press) 